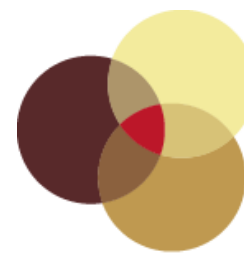

contennporaneity

Vol 7, No 1 (2018) | ISSN 2153-5914 (online) | DOI 10.5195/contemp/2018.269

http://contemporaneity.pitt.edu

\title{
Looking/Not Looking
}

Aaron Henderson

\section{About the Artist}

Aaron Henderson's photographs, videos, and installations are inspired by the artist's visceral and intellectual interest in how the body moves through space. His projects articulate different facets of an ongoing critique of society's relationship to spectacle, technology, and performance. He believes that it is only thorough the process of examination-of ourselves and of our culture-that the past, present, and future can be fully understood.

Well-acquainted with movement, Henderson threw himself into walls and off platforms for STREB Extreme Action, an acrobatic performance company from 2002 to 2006. His videos and installations have been presented at the Carnegie Museum of Art, the Wexner Center, and many other museums and galleries in the United States, Europe, and Asia. His projection designs have been presented at Lincoln Center, the Andy Warhol Museum, the Institute of Contemporary Art (Boston) as well as at theaters and festivals around the world.

Henderson is currently an associate professor in the Department of Studio Arts at the University of Pittsburgh. 


\title{
Looking/Not Looking
}

\author{
Aaron Henderson
}

My photographic series "Looking/Not Looking" (2018) is a collection of traces, reflections, and shadows captured in the Carnegie Museum of Art's African Art/Art before 1300 galleries. Held apart from the rest of the collection, the corridor that houses these objects is fascinating due to the range of work collected there as well as for the ways this space differs from the rest of the museum. In my own work, I often set rigorous and specific parameters. Sometimes these rules are attempts to simplify complex situations, allowing me to focus on one aspect of a larger whole. Sometimes they are just games I play. In this instance, I knew that I wanted to avoid photographing the actual objects, instead focusing on the space itself. As I entered the galleries, I found myself struck by the beauty of the objects and the density of their display. Darker than the surrounding galleries, reflections and shadows dominate the space. The objects themselves seem to glow, with spotlights bouncing off their surfaces and glass encasing. Transparent cubes create structures that contain and protect the objects, which sit on nearly identical pedestals. Scuffs and fingerprints remind us that these are real spaces, despite the austere backgrounds. These photographs look around and between the objects, focusing on the space itself. They examine a gallery tucked away, but only capture glances of the treasures held within.

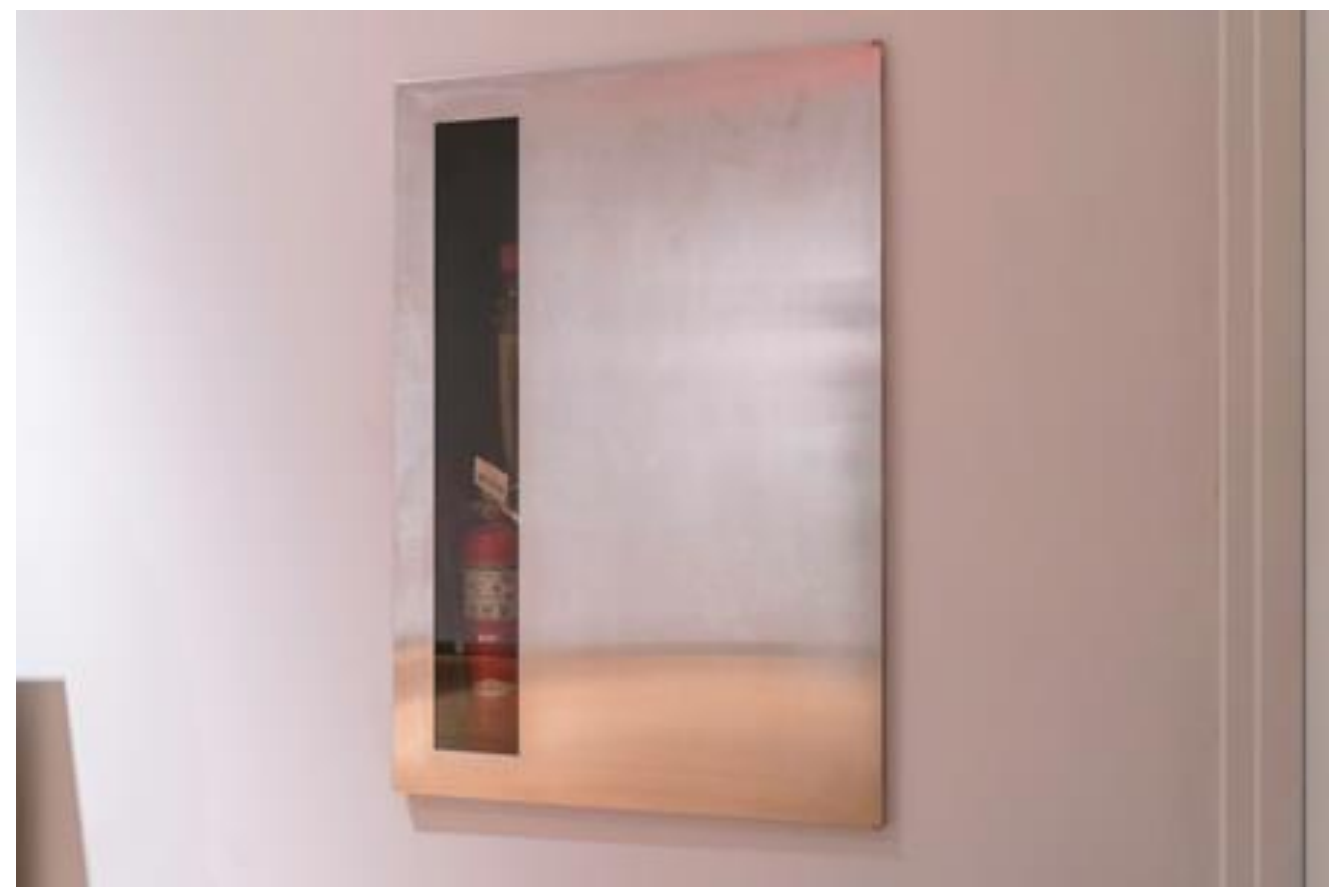

Figure 1

Aaron Henderson, Not Looking 4, 2018, Digital Photograph. 


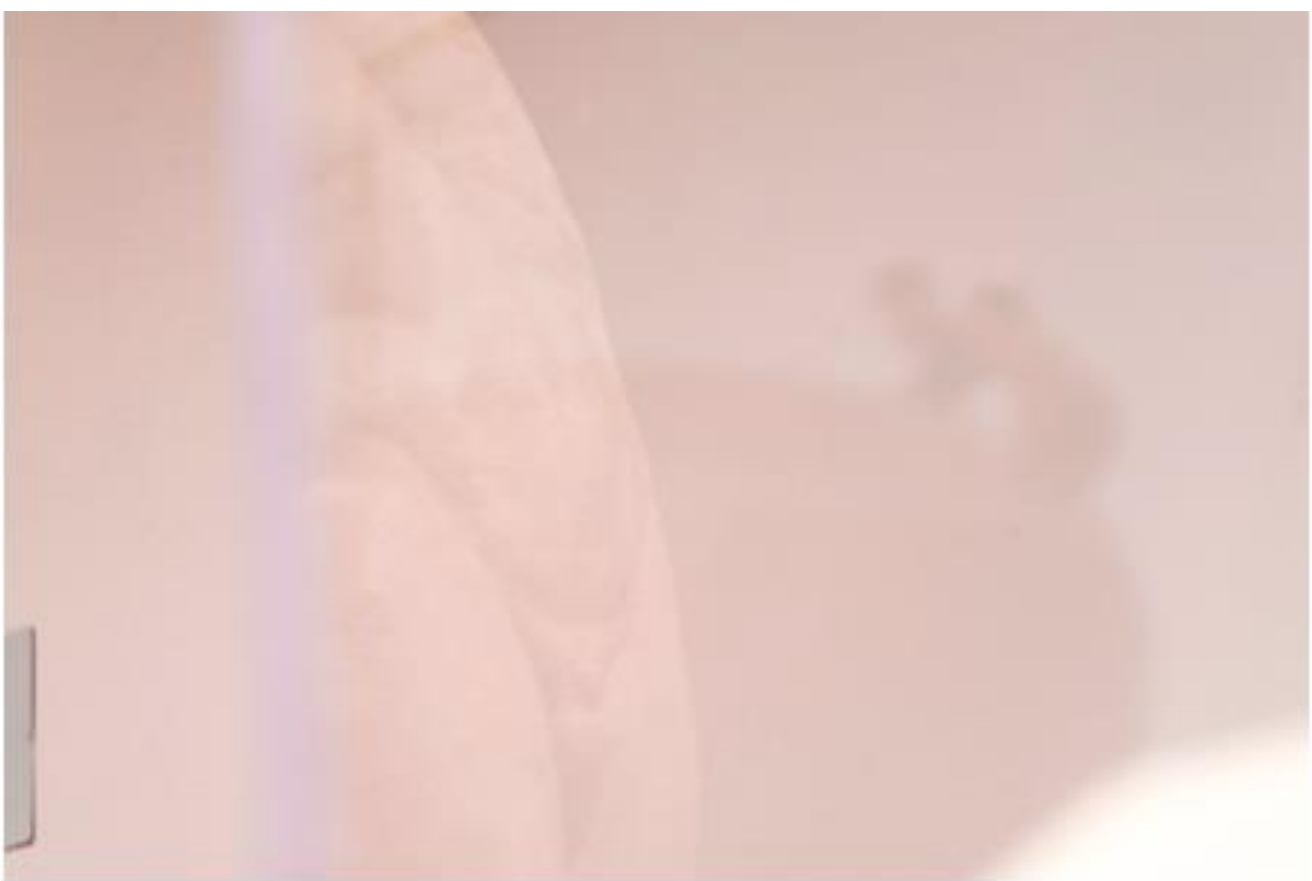

Figure 2

Aaron Henderson, Not Looking 9, 2018, Digital Photograph.

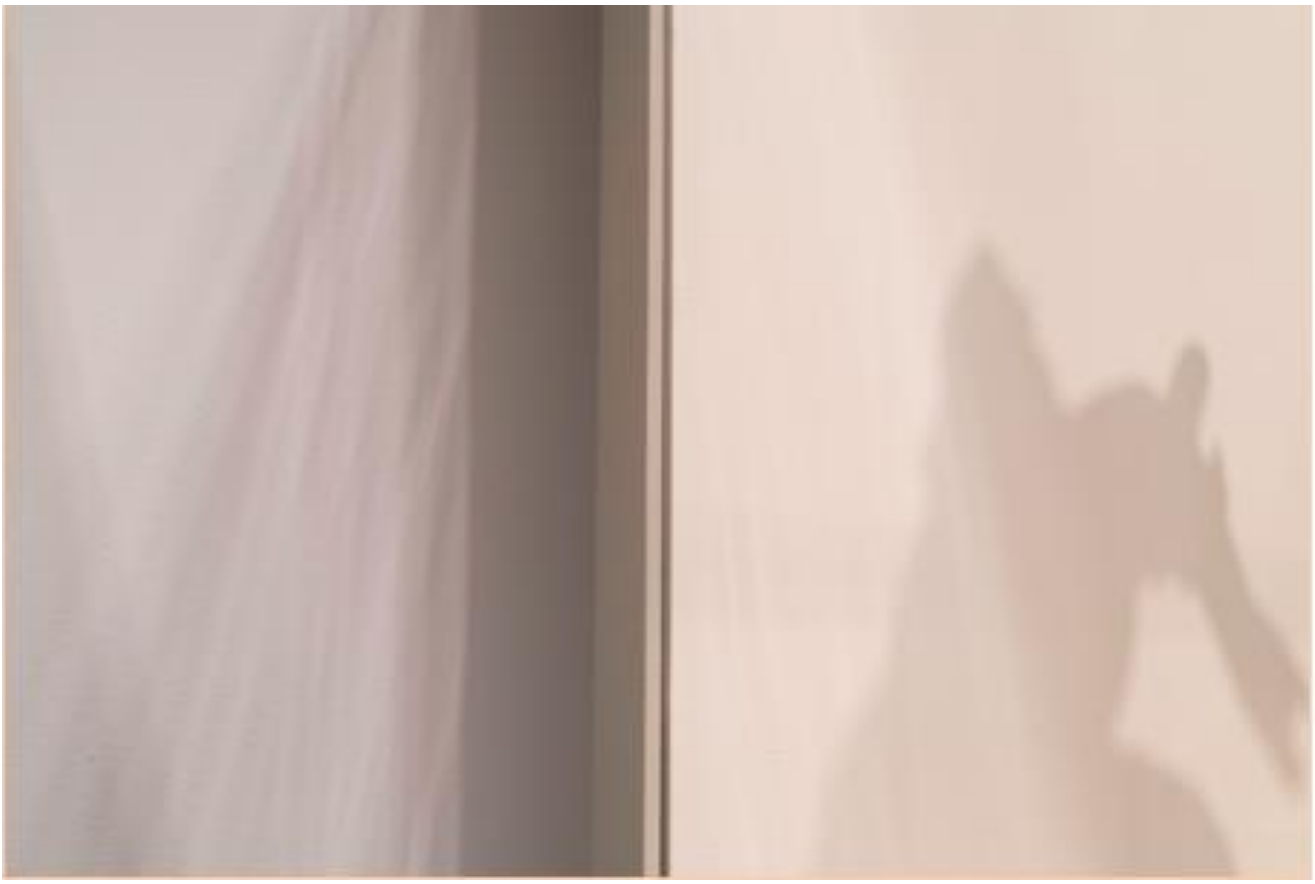

Figure 3

Aaron Henderson, Not Looking 10, 2018, Digital Photograph. 


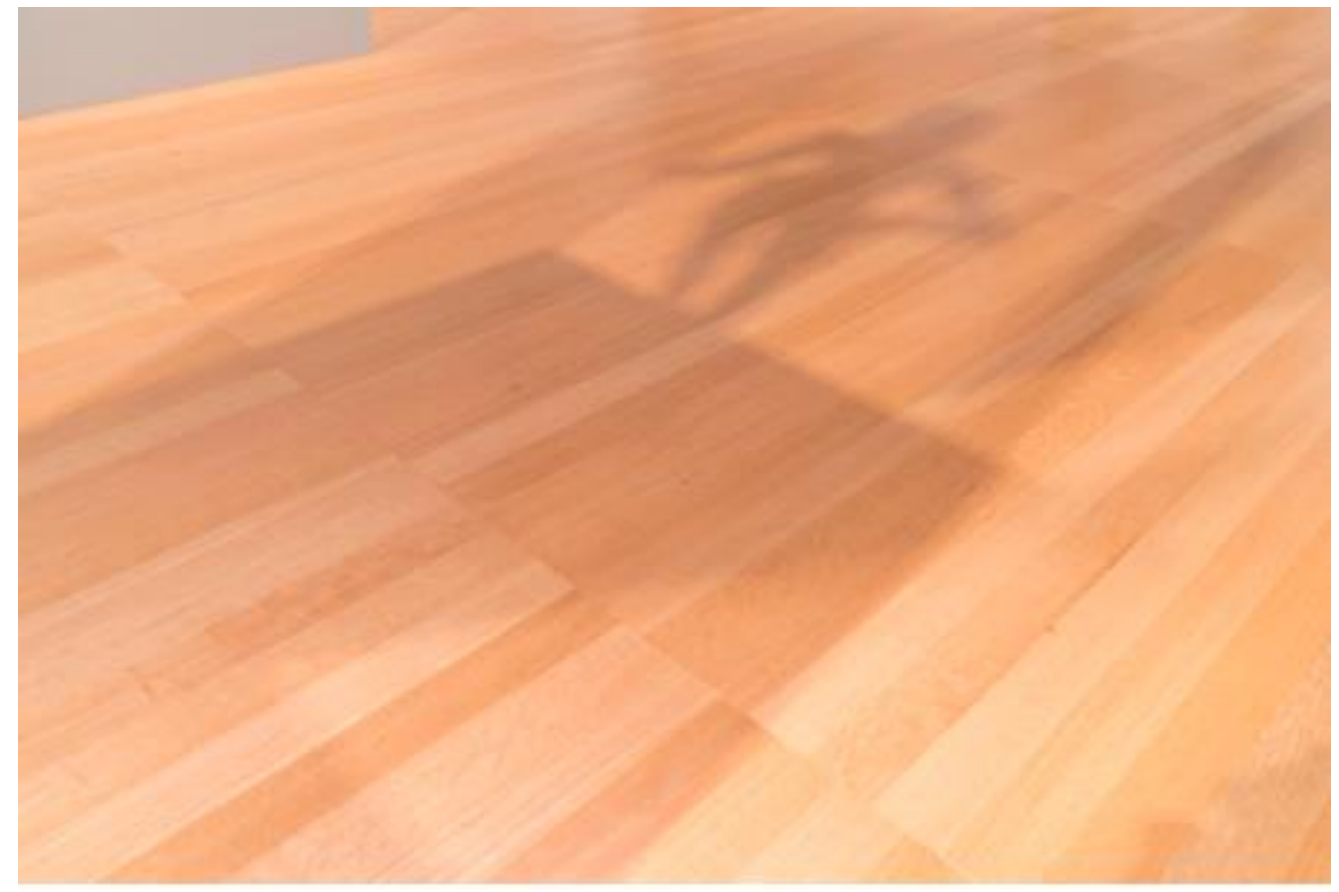

Figure 4

Aaron Henderson, Not Looking 14, 2018, Digital Photograph. Attribution 4.0 United States License.

\section{ULLS D-Sorke}

This journal is operated by the University Library System of the University of Pittsburgh as part of its D-Scribe Digital Publishing Program, and is co-sponsored by the University of Pittsburgh Press. 